\title{
EVALUACIÓN COSTO-EFECTIVIDAD DE DOS ALTERNATIVAS DE VACUNACIÓN PARA EL VIRUS DEL PAPILOMA HUMANO EN LA PREVENCIÓN DEL CÁNCER CERVICAL UTERINO
}

\author{
Rafael Bolaños-Díaz ${ }^{1,2, a, b, c}$, Romina A. Tejada1,3,a,b, Jessica Beltrán ${ }^{1, a}$, Seimer Escobedo-Palza ${ }^{4, a}$
}

\begin{abstract}
RESUMEN
Objetivos. Determinar la relación costo-efectividad de la vacunación contra el (virus del papiloma humano) VPH y el tamiz de lesiones cervicales, frente a un programa de tamiz solo. Materiales y métodos. Se realizó una evaluación costoefectividad y se empleó un modelo de Markov, con un horizonte temporal de 70 años y tres alternativas de prevención para el (cáncer del cuello uterino) CCU (tamiz solo, tamiz + vacuna bivalente, y tamiz + vacuna cuadrivalente), en una cohorte hipotética de niñas de diez años, desde la perspectiva del Ministerio de Salud. Resultados. La vacunación contra el VPH y tamiz es más costo-efectiva que el tamiz solo a partir de una voluntad de pago de S/ 2000 (USD 1290,32). En el análisis determinístico, la vacuna bivalente es marginalmente más costo-efectiva que la vacuna cuadrivalente (S/ 48 [USD 30,97] frente a S/ 166 [USD 107,10] por AVAC, respectivamente). Sin embargo, en el análisis probabilístico ambas intervenciones generan nubes de puntos superpuestos, con una tendencia de la vacuna cuadrivalente a ser más costo-efectiva. Es decir, ambas son costo-efectivas y, por ende, intercambiables. El modelo fue especialmente sensible a variaciones de la cobertura y en la prevalencia de infección persistente por genotipos oncológicos no incluidos en la vacuna. Conclusiones. A partir de una disponibilidad de pago de S/ 2000 [USD 1 290,32] el tamiz y la vacunación son más costo-efectivos que el tamiz solo. La diferencia de costo-efectividad entre ambas vacunas carece de robustez probabilística y ambas vacunas pueden considerarse intercambiables desde la perspectiva costo-efectividad.
\end{abstract}

Palabras clave: Vacunas contra papillomavirus; Neoplasias del cuello uterino; Condiloma acuminado; Análisis costobeneficio (Fuente: DeCS BIREME).

\section{EVALUATION OF THE COST-EFFECTIVENESS OF TWO ALTERNATIVE HUMAN PAPILLOMAVIRUS VACCINES AS PROPHYLAXIS AGAINST UTERINE CERVICAL CANCER}

\begin{abstract}
Objectives. To determine the cost-effectiveness of human papillomavirus (HPV) vaccination and cervical lesion screening versus screening alone for the prevention of uterine cervical cancer (UCC). Materials and methods. This cost-effectiveness evaluation from the perspective of the Ministry of Health employed a Markov model with a 70-year time horizon and three alternatives for UCC prevention (screening alone, screening + bivalent vaccine, and screening + quadrivalent vaccine) in a hypothetical cohort of 10-year-old girls. Results. Our model, which was particularly sensitive to variations in coverage and in the prevalence of persistent infection by oncologic genotypes not included in the vaccine, revealed that HPV vaccination and screening is more cost-effective than screening alone, assuming a payment availability from S/ 2000 (US dollars (USD) 1 290.32) per subject. In the deterministic analysis, the bivalent vaccine was marginally more cost-effective than the quadrivalent vaccine (S/ 48 [USD 30.97] vs. S/ 166 [USD 107.10] per quality-adjusted life-year, respectively). However, in the probabilistic analysis, both interventions generated clouds of overlapping points and were thus cost-effective and interchangeable, although the quadrivalent vaccine tended to be more cost-effective. Conclusions. Assuming a payment availability from S/ 2000 [USD 1,290.32], screening and vaccination were more cost-effective than screening alone. The difference in cost-effectiveness between the two vaccines lacked probabilistic robustness, and therefore the vaccines can be considered interchangeable from a cost-effectiveness perspective.
\end{abstract}

Key words: Papillomavirus vaccines; Cervical intraepithelial neoplasia; Condylomata acuminata; Cost-benefit analysis (Source: MeSH NLM).

\footnotetext{
Unidad de Análisis y Generación de Evidencias en Salud Pública. Centro Nacional de Salud Pública. Instituto Nacional de Salud. Lima, Perú Organización Médica para el desarrollo de la Salud. Lima, Perú.

Escuela de Medicina, Universidad Peruana de Ciencias Aplicadas. Lima, Perú.

Sociedad Peruana de Administración de Salud. Lima, Perú.

Médico cirujano; ${ }^{\mathrm{b}}$ magíster en Epidemiología clínica; ${ }^{\mathrm{c}}$ magíster en Economía de la Salud

Recibido: 21/10/2015 Aprobado: 06/07/2016
}

Citar como: Bolaños-Díaz R, Tejada RA, Beltrán J, Escobedo S. Evaluación costo-efectividad de dos alternativas de vacunación para el virus del papiloma humano en la prevención del cáncer cervical uterino. Rev Peru Med Exp Salud Publica. 2016;33(3):411-8. doi: 10.17843/rpmesp.2016.333.2294 


\section{INTRODUCCIÓN}

La infección por el virus del papiloma humano (VPH) es una de las infecciones de transmisión sexual más frecuentes. Ha sido asociada con patologías malignas como neoplasias intraepiteliales (NIC), cáncer anogenital (cérvix, vagina, vulva, pene y ano), y cáncer oral, asociadas a los genotipos de alto riesgo oncológico. Así mismo, ha sido asociada con patologías benignas como las verrugas ano-genitales (VAG) y la papilomatosis laríngea recurrente, producidas principalmente por genotipos de bajo riesgo oncológico ${ }^{(1-3)}$.

Se ha reportado que el $10 \%$ de mujeres en población general tienen una infección genital por el $\mathrm{VPH}{ }^{(4)}$, y entre ellas, al menos, el $14 \%$ tiene una infección con genotipos de alto riesgo oncológico ${ }^{(5)}$. Además, la mayoría de casos ocurren en mujeres jóvenes (menores de 25 años) ${ }^{(6)}$. Se ha estimado, también, que el $99 \%$ de los casos de cáncer de cuello uterino (CCU) tienen presencia de VPH de alto riesgo oncológico ${ }^{(7)}$ y $70 \%$ específicamente de los genotipos 16 y $18{ }^{(8)}$. Además, el $90 \%$ de los casos de VAG se deben a los genotipos 6 y $11^{\left({ }^{(9)} \text {. }\right.}$

Según la Agencia Internacional para la Investigación en Cáncer, el CCU es el cuarto cáncer más frecuente en mujeres a nivel mundial, habiéndose estimado para el 2012 unos 528000 casos nuevos y 266000 muertes asociadas a CCU a nivel mundial. La mayoría de estos casos $(85 \%)$ y muertes $(87 \%)$ se concentran en países en vías de desarrollo ${ }^{(10)}$. Es necesario tener en consideración que no todas las mujeres que presenten una infección por el VPH desarrollaran CCU.

En el caso del Perú, no hemos encontrado en nuestra revisión de la literatura estudios sobre la prevalencia de infección por el VPH a nivel nacional. Existen estudios regionales, como el de Martorell et al. que en el 2012 reportan una prevalencia de infección por el VPH del $43,9 \%$ en mujeres en lquitos, así como prevalencias de infección por genotipos de alto riesgo de $71,9 \%$, y de genotipos de bajo riesgo de $19,3 \%{ }^{(11)}$. También hay estimaciones de la Organización Mundial de la Salud (OMS) en el año 2010, que indican una prevalencia de infección por el VPH 16/18 en mujeres con citología normal de $6,6 \%$ y en mujeres con CCU de $65,9 \%$. Igualmente, estiman una incidencia de CCU de 31,3 por 100 mil mujeres ${ }^{(12)}$. Finalmente, el Sistema Nacional de Vigilancia Epidemiológica ha reportado que los CCU representan el $14,9 \%$ del total de cánceres en el periodo 2006-2011 (13).

Con relación a las VAG, se ha observado un incremento de los casos en el Reino Unido ${ }^{(14)}$. Se han reportado incidencias que van desde 58 hasta 319 casos por 100 000 personas, la mayoría de casos en mujeres ${ }^{(8,15,16)}$.
Es posible que muchas de estas incidencias estén subestimadas, tanto por un inadecuado registro de los casos como por la falta de búsqueda de atención médica en las personas que las padecen. A este problema debemos sumar que las VAG no solo ocasionan malestar físico a las personas que las padecen, sino que también generan alteraciones psicológicas como ansiedad y depresión, y problemas en la autoimagen, la actividad sexual, miedo al desarrollo de cáncer, y la relación de pareja, debido a los riesgos de transmisión ${ }^{(7,17)}$.

Actualmente, están disponibles en el mercado peruano dos vacunas contra la infección por el VPH: la bivalente, que protege contra los genotipos 16 y 18 (Cervivarix $\AA$, GlaxoSmithKline [GSK]) y la cuadrivalente que incluye, además, los genotipos 6 y 11 (Gardasil $囚$, Merck Sharp\&Dhome [MS\&D]). Ambas vacunas son eficaces en la adquisición de inmunidad y en la prevención de infección persistente y lesiones premalignas por los genotipos incluidos ${ }^{(18,19)}$, así como por los no incluidos ${ }^{(20,21)}$. Además, la vacuna cuadrivalente ha demostrado ser eficaz en la prevención de VAG ${ }^{(22)}$. En base a esta evidencia se ha recomendado la inclusión de la vacuna contra el VPH en programas nacionales de vacunación (23).

Siendo el CCU una enfermedad prevalente en el Perú, se propuso incluir a la vacuna contra el VPH en el esquema nacional de vacunación. Sin embargo, dicha decisión debe basarse tanto en estudios de eficacia y efectividad de la vacuna, como en evaluaciones económicas costoefectividad (24). Las evaluaciones costo-efectividad son herramientas importantes para la toma de decisiones en contextos de escasez de recursos. Dichas evaluaciones permitirán estimar el costo incremental y los efectos en la salud de la vacunación nacional con las dos vacunas disponibles combinada con la estrategia actual de tamiz en comparación con el tamiz solamente. El análisis costoefectividad permitirá responder si los efectos adicionales en la salud que resultan de la vacunación justifican el costo adicional asociado, es decir, cuál es la estrategia más adecuada en términos de costo-efectividad.

Existen estudios en otras realidades que han reportado que la vacunación de niñas no infectadas contra el VPH es costo-efectiva (25-27). Sin embargo, no es posible extrapolar los resultados al Perú, ya que dichos estudios se basan en costos y frecuencias de las patologías y sus complicaciones a nivel local. En dicho contexto, el presente análisis fue parte de los estudios que se realizaron para la toma de decisiones sobre la vacunación contra el VPH en el Perú.

Ante ello, el presente estudio buscó determinar la relación de costo-efectividad de un programa de diagnóstico y vacunación contra el VPH, tanto con la vacuna bivalente como la cuadrivalente, frente a un programa de diagnóstico solo. 


\section{MATERIALES Y MÉTODOS}

\section{DISEÑO}

Se realizó una evaluación económica de tipo costoefectividad, adaptando un modelo de Markov de la historia natural de la infección del HPV al contexto peruano. El modelo de Markov incorporó la vacunación y el tamiz. Se siguió una cohorte hipotética de 100000 niñas de diez años de edad hasta los 80 años de vida.

\section{COSTOS}

Después de definir los flujogramas de atención para los distintos escenarios clínicos, según las Guías de Atención del Ministerio de Salud (MINSA) y del Instituto Nacional de Enfermedades Neoplásicas (INEN), se calcularon los costos con la información brindada por los centros de costos correspondientes, y en el caso de los medicamentos, según información proveniente del Observatorio Peruano de Productos Farmacéuticos (http://observatorio.digemid.minsa. gob.pe/) de la Dirección General de Medicamentos, Insumos y Drogas, en ambos casos se emplearon costos unitarios al año 2013. El costo de las vacunas se determinó según información del Fondo Rotatorio de la Organización Panamericana de la Salud. Para los escenarios clínicos que no contaban con Guías de Atención explícitas se procedió a una valoración de expertos clínicos esbozándose posteriormente la estructura de costos correspondiente. La perspectiva fue del financiador (MINSA), por lo que en el análisis solo se incluyeron los costos médicos directos. La metodología empleada para el costeo es la propuesta por el MINSA ${ }^{28)}$. Además, realizamos una ponderación de los costos en base a las diversas alternativas de tratamiento y en los casos que aplicara a los tres niveles de atención del MINSA. Los costos fueron calculados en soles $(S /) y$, posteriormente, fueron convertidos a dólares americanos (USD) según el factor de paridad del poder adquisitivo al 2014 (Purchasing Power Parity [PPP]) y se presentan en la Tabla $1^{(29)}$.

\section{EFECTIVIDAD}

Se consideraron reportes de ensayos clínicos para determinar la eficacia de las vacunas en la prevención de lesiones premalignas y malignas de cuello uterino, así como VAG ${ }^{(30,31)}$. La efectividad se midió en años de vida ajustados a calidad (AVAC). En la Tabla 1 se

Tabla 1. Valores ingresados al modelo de costo-efectividad de dos vacunas contra el virus del papiloma humano en la prevención del cáncer de cuello uterino

\begin{tabular}{|c|c|c|}
\hline Parámetro & Valor & Referencia \\
\hline \multicolumn{3}{|l|}{ Población } \\
\hline Cobertura & $80 \%$ & (32) \\
\hline RR vacuna bivalente en infección por VPH de alto riesgo & 0,016 & (18) \\
\hline RR vacuna bivalente en infección por VPH oncogénico no 16/18 & 0,68 & $(18)$ \\
\hline RR vacuna bivalente en infección persistente por VPH 16/18 & 0,08 & (18) \\
\hline RR vacuna bivalente en infección persistente VPH oncogénico no vacunal & 0,734 & (18) \\
\hline RR vacuna cuadrivalente en infección por VPH de alto riesgo & 0,025 & (19) \\
\hline RR vacuna cuadrivalente en infección por VPH de bajo riesgo & 0,03 & (46) \\
\hline RR vacuna cuadrivalente en infección por VPH oncogénico no 16/18 & 0,76 & (47) \\
\hline RR vacuna cuadrivalente en infección persistente por VPH 16/18 & 0,58 & $(47)$ \\
\hline RR vacuna cuadrivalente en infección persistente por VPH oncogénico no vacunal & 0,84 & (47) \\
\hline AVAC verrugas genitales & 0,91 & (35) \\
\hline AVAC NIC 1-2 & $0,91^{*}$ & (35) \\
\hline AVAC NIC 3 & 0,87 & (35) \\
\hline AVAC FIGO I & 0,76 & (35) \\
\hline AVAC FIGO II-IV & 0,67 & (35) \\
\hline Costo de la vacunación bivalente & 166,44 & \\
\hline Costo de la vacunación cuadrivalente & 175,2 & \\
\hline Costo tamiz & 65,7 & \\
\hline Costo ponderado de VAG (S/) & 166,44 & \\
\hline Costo ponderado de NIC 1-2 (S/) & 203,67 & \\
\hline Costo ponderado de NIC 3 (S/) & 689,85 & \\
\hline Costo ponderado de FIGO I (S/) & 3173,31 & \\
\hline Costo ponderado de FIGO II-IV (S/) & 7143,78 & \\
\hline
\end{tabular}

RR: riesgo relativo; VPH: virus del papiloma humano; NIC: neoplasia intraepitelial cervical; VAG: verrugas anogenitales; AVAC: años de vida ajustados a calidad; FIGO: Federación Internacional de Ginecología y Obstetricia. Los datos de costo provinieron del estudio de costeo previo. * Se asumió que la utilidad de los estadios NIC 1 y 2 sería similar a la reportada para NIC 1. 
pueden apreciar los valores según vacuna y patología que se ingresaron al modelo.

\section{MODELO ECONÓMICO}

Se elaboró un modelo económico de Markov basado en un análisis de decisión, en un horizonte temporal de 70 años, aplicando tres alternativas de prevención para el CCU en una cohorte hipotética de 100000 niñas prepúberes (diez años de edad). Se compararon dos estrategias de vacunación seguida de tamiz con solo tamiz convencional usando costo por AVAC. Las alternativas posibles fueron: 1) tamiz; 2) vacunación bivalente + tamiz; y 3) vacunación cuadrivalente + tamiz. Igualmente, consideramos siete desenlaces excluyentes para cada alternativa estudiada. Estos desenlaces fueron: sano, infección por VPH 6/11, infección por VPH 16/18, infección por VPH 31/33/45/52/58, NIC, CCU, y muerte.

Para la construcción del modelo tuvimos en cuenta los siguientes supuestos: 1) el tamiz se realizó con una prueba para ADN viral (seis veces en el horizonte temporal de 70 años); 2) no hay coinfección por nuevos contactos sexuales; 3 ) la regresión de las lesiones es completa; 4) la eficacia de la vacuna es constante a lo largo del horizonte temporal (sin dosis de refuerzo); 5) los tres estados temporales en infección por VPH16/18 son NIC1, NIC2 y NIC3; 6) no hubo estado temporal en infección por VPH no-vacunal (VPH 31/33/45/52/58); y 7) existe una cobertura de vacunación del $80 \%$. Finalmente, para ajustar los costos y los efectos en salud futuros a los valores y volúmenes presentes aplicamos una tasa de descuento de $3 \%$ tanto a los costos como a las efectividades, haciéndola variar en el análisis de sensibilidad entre $2 \%$ y $6 \%$. Se puede consultar a los autores por mayor información sobre el modelo.

En cuanto a la información epidemiológica, se utilizaron datos locales respecto a prevalencia de infección por VPH de alto y bajo riesgo según grupo de edad ${ }^{(32)}$. De igual modo, se utilizaron datos locales según edad para incidencia de CCU, mortalidad general, y mortalidad

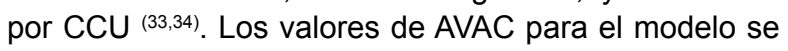
adoptaron de Annemans et al. (35).

Para la evaluación de los resultados se calculó la relación costo-efectividad de cada opción estudiada, considerándose como más eficiente aquella que presente un valor más bajo. También se calcularon los incrementos del costo y la efectividad para las tres alternativas (incremental cost-effectiveness ratio [ICER]), lo que permitió conocer el costo necesario por AVAC para cada una de ellas. Se consideraron ciclos de un año. Finalmente, se realizaron pruebas de sensibilidad determinística y probabilística (1000 simulaciones de Monte Carlo). Se utilizó para el análisis el software TreeAge Pro Version 2014 (TreeAge Software, Inc., Williamstown, MA, USA). EI presente reporte sigue las indicaciones de la Sociedad Internacional de Farmacoeconomía e Investigación de Resultados (ISPOR, por sus siglas en inglés) ${ }^{(36)}$.

\section{CALIBRACIÓN}

Se realizó la calibración del modelo en base al número de casos y muertes estimados en el brazo de la estrategia tamiz, comparándolos con los datos estimados por GLOBOCAN $2012{ }^{(33)}$ y datos de mortalidad por CCU en el 2012 proporcionados por la Dirección General de Epidemiología.

\section{RESULTADOS}

La vacunación contra el VPH y tamiz es más costo-efectiva que el tamiz solo a partir de una voluntad de pago de $S / 2000$ (USD 1 290,32). La intervención con la vacuna cuadrivalente es mínimamente más costosa que con la vacuna bivalente (S/ 3 485,16 [USD 2 248,49] frente a S/ 3 415,55 [USD 2 203,58]) y ligeramente más efectiva $(65,37$ frente a 65,78 AVACs). En el análisis determinístico la vacuna bivalente es marginalmente más costo-efectiva que la vacuna cuadrivalente (S/ 48 [USD 30,97] frente a S/ 166 [USD 107,10] por AVAC, respectivamente) (Tabla 2). Sin embargo, en el análisis probabilístico observamos que ambas intervenciones generan nubes de puntos superpuestos, con una tendencia de la vacuna cuadrivalente a ser más costoefectiva (Figura 1). Es decir, ambas son costo-efectivas $y$, por ende, intercambiables desde la perspectiva costoefectividad.

\section{ANÁLISIS MULTIVARIADO}

En el análisis multivariado (Figura 2) se observó que el modelo era especialmente sensible a variaciones en la

Tabla 2. Análisis incremental determinístico de la vacunación contra el virus del papiloma humano*

\begin{tabular}{lcccccc}
\hline & Costo & $\begin{array}{c}\text { Costo } \\
\text { incremental }\end{array}$ & Efectividad & $\begin{array}{c}\text { Efectividad } \\
\text { incremental }\end{array}$ & C/E & ICER $^{*}$ \\
\hline Tamiz & S/ 3 167,06 & & $60,18 \mathrm{Q}$ & & $\mathrm{S} / 52,63$ & \\
Tamiz + vacuna 2x & $\mathrm{S} / 3418,55$ & $\mathrm{~S} / 251,49$ & $65,37 \mathrm{Q}$ & $5,19 \mathrm{Q}$ & $\mathrm{S} / 52,29$ & $\mathrm{~S} / 48,39 / \mathrm{AVAC}$ \\
Tamiz + vacuna 4x & $\mathrm{S} / 3445,16$ & $\mathrm{~S} / 318,10$ & $65,78 \mathrm{Q}$ & $5,60 \mathrm{Q}$ & $\mathrm{S} / 52,98$ & $\mathrm{~S} / 165,67 / \mathrm{AVAC}$ \\
\hline
\end{tabular}

2x: bivalente; 4x: cuadrivalente. C/E: Costo-efectividad; ICER: razón de incrementos del costo y la efectividad (incremental cost-effectiveness ratio)

* Todas las opciones referidas a una base de línea común. 


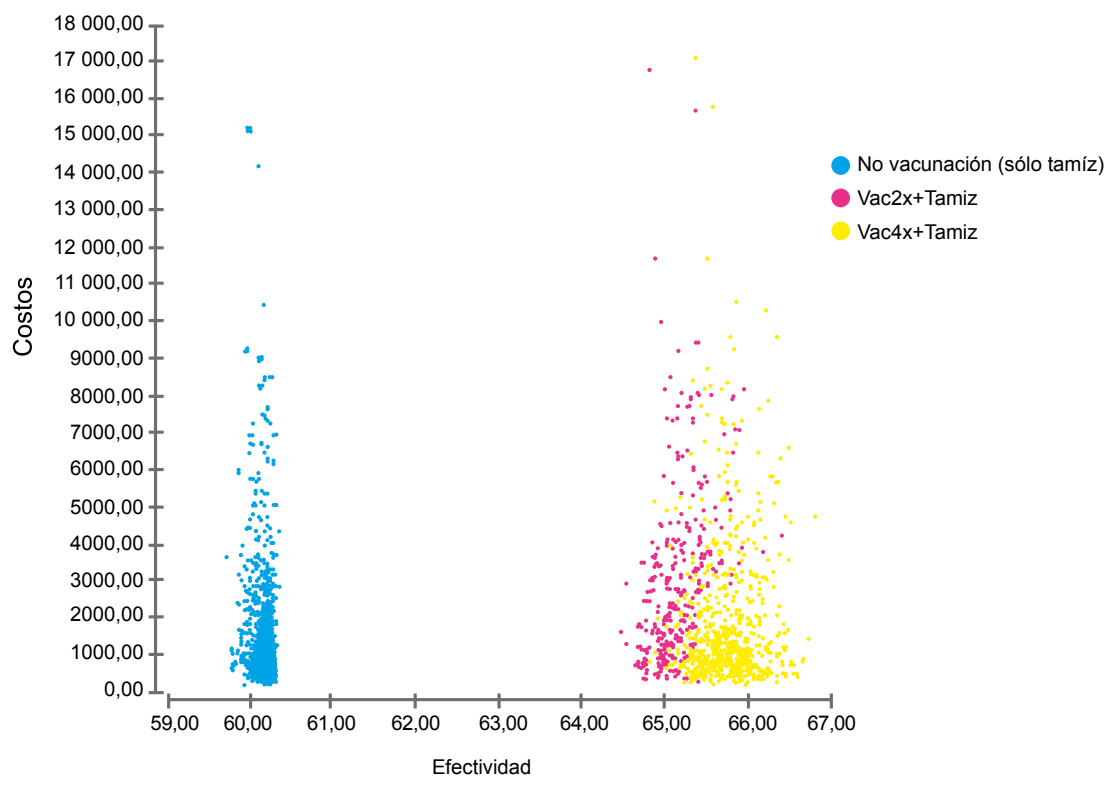

Figura 1. Análisis costo-efectividad probabilístico (simulación de Monte Carlo)

cobertura, y en la prevalencia de infección persistente por genotipos oncológicos no incluidos en la vacuna, así como a variaciones en la prevalencia de infección persistente por los genotipos 16 y 18, prevalencia de $\mathrm{NIC}$, descuento, y prevalencia de VAG. En el análisis de sensibilidad de una vía observamos que en el caso de una cobertura inferior al $65 \%$ la vacuna cuadrivalente quedaba dominada por la bivalente.

\section{CALIBRACIÓN}

Como se observa en la Figura 3, el modelo se acercó a los datos estimados por GLOBOCAN 2012 para Perú con respecto a mortalidad incidental por CCU. Al mismo tiempo, el modelo fue calibrado considerando los datos de la Dirección General de Epidemiología al año 2012. Por ello consideramos que el modelo representa a la realidad peruana.

\section{DISCUSIÓN}

La vacunación contra el VPH es una estrategia muy costo-efectiva en el Perú según las recomendaciones de la OMS ${ }^{(37)}$, ya que su ICER se encuentra por debajo del producto bruto interno (PBI) per cápita (USD 6 600) ${ }^{(38)}$. La diferencia entre ambas vacunas fue marginal, por lo que consideramos que ambas vacunas son equiparables desde la perspectiva de costo-efectividad. El modelo es especialmente sensible a variaciones en la cobertura y en la prevalencia de infección persistente por genotipos oncológicos no incluidos en la vacuna.

Se ha reportado previamente que la vacunación contra el VPH es una estrategia costo-efectiva ${ }^{(39)}$. Se ha reportado también que la vacunación de mujeres tendría un impacto beneficioso en varones debido a la protección comunitaria ${ }^{(25)}$. En relación al tipo de vacuna a emplear, diversos estudios han reportado que la vacuna cuadrivalente es más costo-efectiva que la vacuna bivalente ${ }^{(25,27,40)}$, principalmente debido al efecto a corto plazo sobre las VAG. Debemos resaltar el estudio de Jit et al., en el cual reportan que si bien la vacuna cuadrivalente es más costo-efectiva, las diferencias con la vacuna bivalente serían muy marginales, similar a lo reportado por nosotros ${ }^{(40)}$.

También existen estudios que han reportado a la vacuna bivalente como más costo-efectiva. Sin embargo, consideramos que hay algunas limitaciones en dichos modelos. Por ejemplo, Capri et al. consideraron en el modelo solo los casos incidentes de VAG, por lo que podrían haber subvalorado la carga de enfermedad por dicha patología ${ }^{(41)}$. Igualmente, Lee et al. reportan que la vacuna bivalente es más costo-efectiva cuando la medida de efectividad son años de vida ganados; sin embargo, cuando la medida son AVACs la vacuna cuadrivalente pasa a ser más costo-efectiva ${ }^{(42)}$.

En un escenario donde los recursos son limitados y las necesidades ilimitadas consideramos que los estudios de costo-efectividad son útiles para la toma de decisiones. Otros países se han basado en evaluaciones de este tipo para la toma de decisiones en salud pública; por ejemplo, en el Reino Unido, se decidió cambiar el programa de vacunación nacional de la vacuna bivalente a la cuadrivalente en base a estudios que demostraban que la vacuna cuadrivalente proveía de mejor valor por el dinero que la vacuna bivalente ${ }^{(43)}$.

A nuestro conocer, esta sería la primera evaluación económica en el país que compara ambas vacunas, incluyendo el efecto en las VAG. A diferencia de muchos 


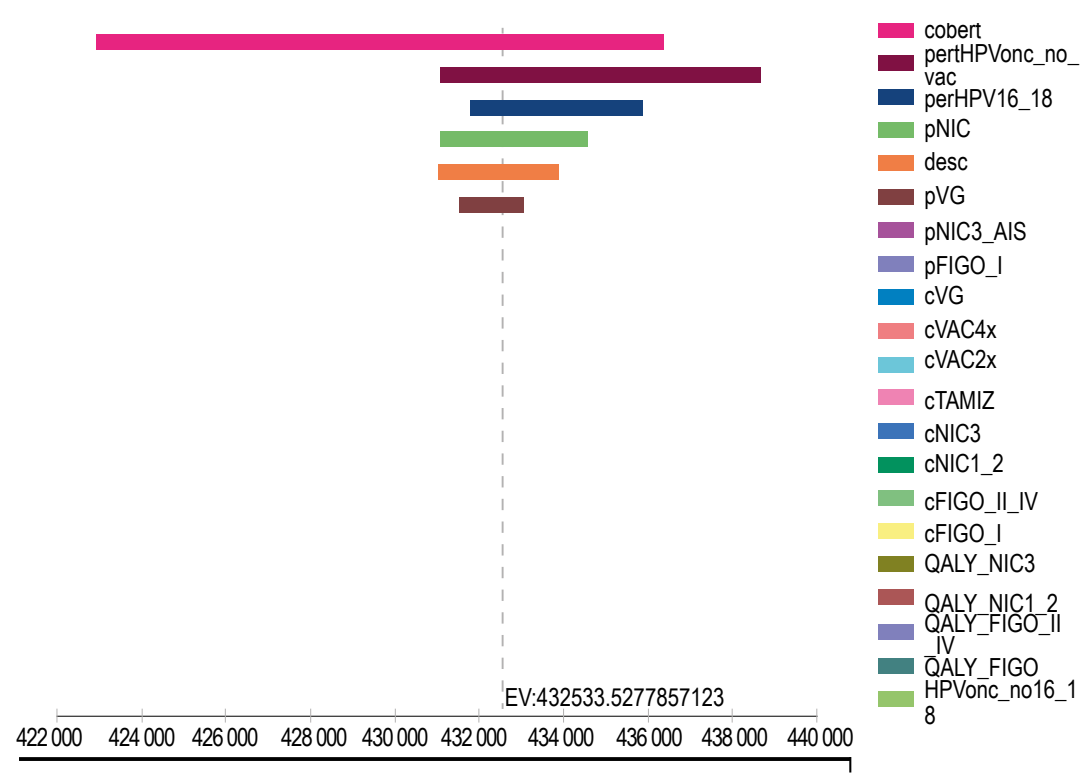

Figura 2. Análisis de tornado del análisis de costo-efectividad de la vacunación contra el VPH en niñas prepúberes

estudios que emplean reportes en la literatura para la estimación de costos, en el presente análisis se emplearon datos recogidos del sistema de salud, lo cual es una fortaleza del estudio.

Dentro de las limitaciones del estudio, en primer lugar, debemos tener en cuenta que el presente modelo solo ha incluido en su análisis las lesiones premalignas y malignas en cuello uterino así como las VAG. No se ha incluido el efecto de la vacunación en otros tipos de cáncer distintos al CCU como el cáncer de vulva y vagina, debido a que solo existen datos provenientes de ensayos clínicos sobre la eficacia de la vacuna cuadrivalente en dichos desenlaces, mas no de la vacuna bivalente ${ }^{(44)}$. Del mismo modo, no hay estudios sobre el potencial efecto de las vacunas contra el VPH en cáncer de ano u orofaringe, ni en papilomatosis laríngea recurrente. Sin embargo, teniendo en consideración que estas son patologías infrecuentes, consideramos que su inclusión no alteraría el modelo.

En segundo lugar, se debe considerar que la frecuencia de VAG proviene de un estudio realizado a nivel de servicios de salud, y en la literatura se ha reportado que existe un subreporte a dicho nivel (16). Tampoco hemos considerado el impacto de la vacunación contra el VPH en varones, debido a una inmunidad comunitaria a través de la vacunación de mujeres ("efecto rebaño"). Otros estudios han reportado que la vacunación de varones no sería costo-efectiva ${ }^{(26)}$. Finalmente, no hay datos

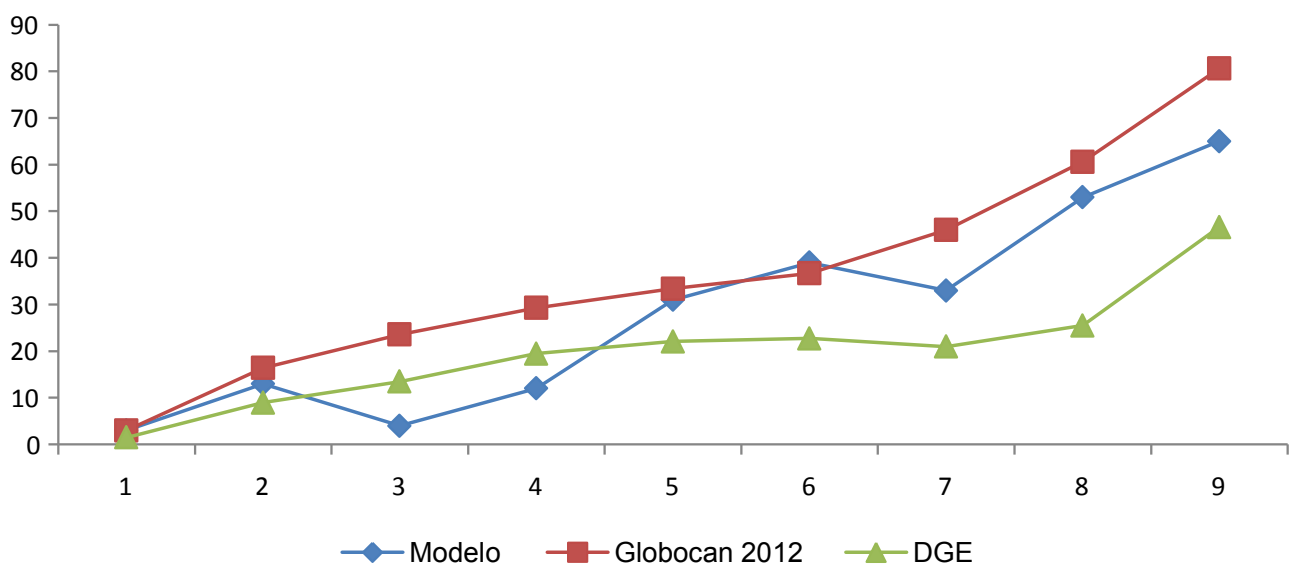

DGE: Dirección General de Epidemiología

Figura 3. Calibración del modelo en base a muertes incidentales por cáncer de cuello uterino (100 000 mujeres-año) 
suficientes para definir mayores desenlaces en el estado de infección por virus no-vacunal (VPH 33/33/45/52/58).

En conclusión, a partir de una disponibilidad de pago de S/ 2000 [USD 1 290,32], tamizar y vacunar es más costo-efectivo que tamizar únicamente. La diferencia de costo-efectividad entre ambas vacunas carece de robustez probabilística y ambas vacunas pueden considerarse intercambiables desde la perspectiva costo-efectividad. Recientemente ha sido aprobada en Estados Unidos la vacuna nonavalente, por lo que consideramos que en un futuro sería necesario actualizar el modelo incluyendo esta opción ${ }^{(45)}$. Las actividades de diagnóstico y tratamiento temprano deben continuar de manera conjunta con las campañas de vacunación, ya que debemos recordar que el $30 \%$ de casos de CCU se encuentran asociados a genotipos no incluidos en las vacunas.

Contribuciones de autoría: RBD y RAT han participado en la concepción y diseño del artículo, RBD, RAT, JB y SE han participado en la recolección, análisis e interpretación de datos. RBD y RAT elaboraron el primer borrador del artículo el cual fue revisado, mejorado y aprobado por todos los autores.

Fuente de financiamiento: Instituto Nacional de Salud.

Conflictos de interés: los autores declaran no tener conflictos de interés.

\section{REFERENCIAS BIBLIOGRÁFICAS}

1. Palefsky JM. Human papillomavirusrelated disease in men: not just a women's issue. J Adolesc Health. 2010;46(4 Suppl):S12-9. doi: 10.1016/j. jadohealth.2010.01.010.

2. Jablonska S, Majewski S. Human papillomavirus infection in women. Special aspects of infectious diseases in women. Clin Dermatol. 1997;15(1):6779.

3. Nyitray AG, Iannacone MR. The epidemiology of human papillomaviruses. Curr Probl Dermatol. 2014;45:75-91. doi: $10.1159 / 000358370$.

4. de Sanjosé S, Diaz M, Castellsagué X, Clifford G, Bruni L, Muñoz N, et al. Worldwide prevalence and genotype distribution of cervical human papillomavirus DNA in women with normal cytology: a meta-analysis. Lancet Infect Dis. 2007;7(7):453-9.

5. Revzina NV, Diclemente RJ. Prevalence and incidence of human papillomavirus infection in women in the USA: a systematic review. Int J STD AIDS. 2005;16(8):528-37.

6. Smith JS, Melendy A, Rana RK, Pimenta JM. Age-specific prevalence of infection with human papillomavirus in females: a global review. J Adolesc Health. 2008;43(4 Suppl):S5-25, S25.e1-41. doi: 10.1016/j. jadohealth.2008.07.009.

7. Maw RD, Reitano M, Roy M. An international survey of patients with genital warts: perceptions regarding treatment and impact on lifestyle. Int J STD AIDS. 1998;9(10):571-8.

8. Hartwig $S$, Syräänen $S$, Dominiak-Felden G, Brotons M, Castellsagué X. Estimation of the epidemiological burden of human papillomavirus-related cancers and nonmalignant diseases in men in Europe: a review. BMC Cancer. 2012;12:30. doi:

\subsection{6/1471-2407-12-30.}

9. Brown DR, Schroeder JM, Bryan JT, Stoler $\mathrm{MH}$, Fife KH. Detection of multiple human papillomavirus types in Condylomata acuminata lesions from otherwise healthy and immunosuppressed patients. J Clin Microbiol. 1999;37(10):3316-22.

10. International Agency for Research on Cancer, World Health Organization. Globocan 2012: Estimated cancer incidence, mortality and prevalence worldwide in 2012. Cervix uteri [internet]. Lyon: IARC/WHO; 2012 [citado el 7 de enero de 2015]. Disponible en: http://globocan.iarc.fr/Pages/fact sheets_cancer.aspx

11. Martorell M, Garcia-Garcia JA, GomezCabrero D, Del Águila A. Comparison of the prevalence and distribution of human papillomavirus infection and cervical lesions between urban and native habitants of an Amazonian region of Peru. Genet Mol Res. 2012;11(3):2099-106. doi: 10.4238/2012.August.6.14.

12. Bruni L, Barrionuevo-Rosas L, Albero G, Aldea M, Serrano B, Valencia S, et al. ICO Information Centre on HPV and Cancer (HPV Information Centre). Human Papillomavirus and Related Diseases in the World. Summary Report 2014-12-18 [Internet]. Barcelona: ICO; 2014 [citado el 14 de enero de 2015]. Disponible en: http://www.hpvcentre.net/statistics/ reports/XWX.pdf

13. Dirección General de Epidemiología. Perú, Ministerio de Salud. Análisis de la situación del cáncer en el Perú 2013. Lima: DGE/MINSA; 2013.

14. Simms I, Fairley CK. Epidemiology of genital warts in England and Wales: 1971 to 1994. Genitourin Med. 1997;73(5):365-7.
15. Scarbrough Lefebvre CD, Van Kriekinge G, Gonçalves MA, De Sanjose S. Appraisal of the burden of genital warts from a healthcare and individual patient perspective. Public Health. 2011;125(7):464-75. doi: 10.1016/j. puhe.2011.01.016.

16. Patel H, Wagner M, Singhal P, Kothari S. Systematic review of the incidence and prevalence of genital warts. BMC Infect Dis. 2013;13:39. doi: 10.1186/14712334-13-39.

17. Filiberti A, Tamburini M, Stefanon B, Merola M, Bandieramonte G, Ventafridda $\mathrm{V}$, et al. Psychological aspects of genital human papillomavirus infection: a preliminary report. J Psychosom Obstet Gynaecol. 1993;14(2):145-52.

18. Paavonen J, Naud P, Salmerón J, Wheeler CM, Chow SN, Apter D, et al. Efficacy of human papillomavirus (HPV)-16/18 AS04-adjuvanted vaccine against cervical infection and precancer caused by oncogenic HPV types (PATRICIA): final analysis of a double-blind, randomised study in young women. Lancet. 2009:374(9686):301-14. doi: 10.1016/ S0140-6736(09)61248-4.

19. FUTURE II Study Group. Quadrivalent vaccine against human papillomavirus to prevent high-grade cervical lesions. $\mathrm{N}$ Engl J Med. 2007;356(19):1915-27.

20. Wheeler CM, Castellsagué X, Garland SM, Szarewski A, Paavonen J, Naud P, et al. Cross-protective efficacy of HPV16/18 AS04-adjuvanted vaccine against cervical infection and precancer caused by non-vaccine oncogenic HPV types: 4-year end-of-study analysis of the randomised, double-blind PATRICIA trial. Lancet Oncol. 2012;13(1):100-10. doi: 10.1016/S1470-2045(11)70287-X. 
21. Wheeler CM, Kjaer SK, Sigurdsson K, Iversen OE, Hernandez-Avila M, Perez $\mathrm{G}$, et al. The impact of quadrivalent human papillomavirus (HPV; types 6, 11,16 , and 18) L1 virus-like particle vaccine on infection and disease due to oncogenic nonvaccine HPV types in sexually active women aged $16-26$ years. J Infect Dis. 2009;199(7):936-44. doi: $10.1086 / 597309$.

22. Muñoz N, Kjaer SK, Sigurdsson $K$, Iversen O-E, Hernandez-Avila $\mathrm{M}$ Wheeler CM, et al. Impact of human papillomavirus (HPV)-6/11/16/18 vaccine on all $\mathrm{HPV}$-associated genital diseases in young women. J Natl Cancer Inst. 2010;102(5):325-39. doi: 10.1093/ jnci/djp534.

23. Crosignani P, De Stefani A, Fara GM, Isidori AM, Lenzi A, Liverani CA, et al. Towards the eradication of HPV infection through universal specific vaccination. BMC Public Health. 2013;13:642. doi: 10.1186/1471-2458-13-642.

24. Ciliska D, Thomas H, Buffett C. An Introduction to Evidence-Informed Public Health and A Compendium of Critical Appraisal Tools for Public Health Practice. Hamilton, ON: National Collaborating Centre for Methods and Tools; 2012. p. 27.

25. Bresse X, Adam M, Largeron N, Roze S, Marty R. A comparative analysis of the epidemiological impact and disease costsavings of HPV vaccines in France. Hum Vaccines Immunother. 2013;9(4):82333. doi: $10.4161 / \mathrm{hv} .22994$.

26. Jit M, Choi YH, Edmunds WJ. Economic evaluation of human papillomavirus vaccination in the United Kingdom. BMJ. 2008;337:a769. doi: 10.1136/bmj.a769.

27. Aponte-González J, Fajardo-Bernal L, Diaz J, Eslava-Schmalbach J, Gamboa O, Hay JW. Cost-effectiveness analysis of the bivalent and quadrivalent human papillomavirus vaccines from a societal perspective in Colombia. PloS One. 2013;8(11):e80639. doi: 10.1371/ journal.pone.0080639.

28. Seguro Integral de Salud. Perú, Ministerio de Salud. Directiva Administrativa 004-2012-SIS-GREP. Directiva que norma el proceso de "Evaluación de tecnologías sanitarias" del Seguro Integral de Salud. Lima: SIS/MINSA; 2012.

29. The World Bank. PPP conversion factor, GDP (LCU per international \$) [Internet]. Washington, D.C.: The World Bank; 2016 [citado el 31 de mayo de 2016]. Disponible en: http://data. worldbank.org/indicator/PA.NUS.PPP/ countries?display $=$ default

30. Tejada R, Vargas K, Benites-Zapata V, Mezones-Holguín E, Bolaños-Díaz $\mathrm{R}$, Fiestas F, et al. Eficacia de la vacuna contra el virus del papiloma humano en la prevención de lesiones no oncológicas: revisión sistemática y meta-análisis. Lima: UNAGESP/INS; 2014. p. 41.

31. Bolaños-Díaz R, Mezones-Holguín E, Fiestas F, Suarez V. Eficacia de las vacunas bivalente y cuadrivalente del papilomavirus humano (VPH) para prevenir lesiones cervico-uterinas premalignas o malignas: revisión sistemática. Lima: UNAGESP/INS; 2012.

32. Goldie SJ, Levin C, Mosqueira-Lovón NR, Ortendahl J, Kim J, O'Shea M, et al. Health and economic impact of human papillomavirus 16 and 18 vaccination of preadolescent girls and cervical cancer screening of adult women in Peru. Rev Panam Salud Publica. 2012;32(6):42634.

33. International Agency for Research on Cancer, World Health Organization. Globocan 2012: Estimated cancer incidence, mortality and prevalence worldwide in 2012 [internet]. Lyon: IARC/WHO; 2012 [citado el 16 de junio de 2014]. Disponible en: http:// globocan.iarc.fr/Pages/fact_sheets_ cancer.aspx

34. GBD Compare [Internet]. Washington, D.C.: Institute for Health Metrics and Evaluation. c2013- [citado el 12 de junio de 2014]. Disponible en: http://viz. healthmetricsandevaluation.org/gbdcompare/

35. Annemans L, Rémy V, Oyee J, Largeron N. Cost-effectiveness evaluation of a quadrivalent human papillomavirus vaccine in Belgium. PharmacoEconomics. 2009;27(3):23145. doi: 10.2165/00019053-20092703000006.

36. Husereau D, Drummond M, Petrou S, Carswell C, Moher D, Greenberg $\mathrm{D}$, et al. Consolidated Health Economic Evaluation Reporting Standards (CHEERS)--explanation and elaboration: a report of the ISPOR Health Economic Evaluation Publication Guidelines Good Reporting Practices Task Force. Value Health. 2013;16(2):231-50. doi: 10.1016/j. jval.2013.02.002.

37. World Health Organization. Making choices in health: Who guide to costeffectiveness analysis. Geneva: WHO; 2003. p. 329.

38. Banco Mundial. PIB per cápita (US\$ a precios actuales) [Internet]. Washington, D.C.: Banco Mundial. 2014 [citado el 25 de noviembre de 2014]. Disponible en: http://datos. bancomundial.org/indicador/NY.GDP. PCAP.CD ?order $=$ wbapi data value_2013+wbap i_data_value+wbapi_ data_value-last\&sort $=$ asc

39. Obradovic M, Mrhar A, Kos M. Costeffectiveness analysis of $\mathrm{HPV}$ vaccination alongside cervical cancer screening programme in Slovenia Eur J Public Health. 2010;20(4):415-21. doi: 10.1093/eurpub/ckp208.

40. Jit M, Chapman R, Hughes O, Choi YH. Comparing bivalent and quadrivalent human papillomavirus vaccines: economic evaluation based on transmission model. BMJ. 2011;343:d5775. doi: 10.1136/bmj. d5775.

41. Capri S, Gasparini R, Panatto D, Demarteau N. Cost-consequences evaluation between bivalent and quadrivalent $\mathrm{HPV}$ vaccines in Italy: the potential impact of different crossprotection profiles. Gynecol Oncol. 2011;121(3):514-21. doi: 10.1016/j. ygyno.2011.01.029.

42. Lee VJ, Tay SK, Teoh YL, Tok MY. Cost-effectiveness of different human papillomavirus vaccines in Singapore. BMC Public Health. 2011;11:203. doi: 10.1186/1471-2458-11-203

43. Health Protection Agency. Human papillomavirus (HPV) - cervical cancer and genital warts [Internet]. London: HPA; 2014 [citado el 21 de julio de 2014]. Disponible en: http://www. hpa.org.uk/Topics/InfectiousDiseases/ InfectionsAZ/GenitalWarts/

44. Kjaer SK, Sigurdsson K, Iversen O-E, Hernandez-Avila $M$, Wheeler CM, Perez G, et al. A pooled analysis of continued prophylactic efficacy of quadrivalent human papillomavirus (Types 6/11/16/18) vaccine against high-grade cervical and external genital lesions. Cancer Prev Res (Phila). 2009;2(10):868-78. doi: 10.1158/1940 6207.CAPR-09-0031.

45. Joura EA, Giuliano AR, Iversen O-E, Bouchard C, Mao C, Mehlsen J, et al. A 9-valent HPV vaccine against infection and intraepithelial neoplasia in women. N Engl J Med. 2015;372(8):711-23. doi: 10.1056/NEJMoa1405044.

46. Garland SM, Hernandez-Avila M, Wheeler CM, Perez G, Harper DM, Leodolter $S$, et al. Quadrivalent vaccine against human papillomavirus to prevent anogenital diseases. N Engl J Med. 2007 May 10;356(19):1928-43.

47. Brown DR, Kjaer SK, Sigurdsson K, Iversen O-E, Hernandez-Avila $M$, Wheeler CM, et al. The impact of quadrivalent human papillomavirus (HPV; types 6, 11, 16, and 18) L1 viruslike particle vaccine on infection and disease due to oncogenic nonvaccine HPV types in generally HPV-naive women aged 16-26 years. J Infect Dis. 2009;199(7):926-35.

Correspondencia: Rafael Bolaños-Diaz Correoelectrónico:rbolanosd@yahoo.es Dirección: Av. Defensores del Morro 2268 (Ex Huaylas) Chorrillos, Lima 9, Perú Teléfono: 7481111 Anexo 6650 